\title{
An Efficient Energy Measurement and Control using IoT
}

\author{
Vijaya Anand Nidumolu, Venu Madhav Panchagnula, G.L.N. Murthy
}

\begin{abstract}
This paper presents a new hardware and web-based Energy Measurement \& control system for Internet of Things (IoT) platform. The appliances can share information and communication with each other through web. It helps in analyze and control of the appliances anytime and anywhere from the world. The desired objective of remote monitoring and centralized management through IoT technology are achieved, it enables flexible control mechanism through a secured internet connection. This revolutionary model helps in the design of hardware components for developments With IoT, we can convert physical components like machines and appliances which are used in day to day life into smart appliances.
\end{abstract}

Keywords: Energy Management, IOT, Smart Technology, Arduino.

\section{INTRODUCTION}

The residential power distribution network is becoming a more complex network with the increased demand. Energy management plays a vital role in defining the position of the domestic consumers in the power network. By updating towards the IT-based devices such as smart meters, smart appliances in home, converts the residential consumer from passive mode to active mode. In addition, it also helps in measuring the modern electricity for over all time of consumption and critical peak period consumption apart from real rime pricing of customers for managing the loads in prospect of both financial and environmental point of view. Thus, the Energy Measurement System (EMS), acts as the 'manager' in the smart homes and buildings, EMS includes planning and operation of consumption with main objectives as resource conservation, and cost savings, allowing the users have permanent access to the energy they need. The reason why one should go for EMS is to be energy-conscious and make every exertion to save electricity. Conservation can save the money.

Revised Manuscript Received on December 30, 2019.

* Correspondence Author

Vijaya anand Nidumolu*, Electrical and Electronics Eng. Department, P.V.P Siddhartha Institute of technology, Vijayawada, India.

Venu Madhav P, Electronics and Communication Eng. Department, P.V.P Siddhartha Institute of technology, Vijayawada, India.

GLN Murthy, Electronics and Communication Eng. Department, L. B. R. College of Engineering, City, Mylavaram, India.

(C) The Authors. Published by Blue Eyes Intelligence Engineering and Sciences Publication (BEIESP). This is an open access article under the CC-BY-NC-ND license http://creativecommons.org/licenses/by-nc-nd/4.0/
Because of which many started to habituate a culture of conservation. Lesser the usage of electricity means lesser fossil fuels burned. Indeed, we can also generate electricity from renewable sources of energy such as wind and solar, but much of the electrical energy that we consume is still from sources such as oil and coal. The more electrical energy we consume the faster these resources deplete being a non-renewable source. Fossil fuels are not a clean source of energy either. Hence, conservation of electrical energy leads to less pollution and reduce carbon emission gas. Few of the challenges that are faced by today's EMS are numerous metering points, distributed metering points, delayed levels of automation. Over the past few decades the EMS is acquiring the information artificially leading to drawbacks such as little information, lower transmission rate, lengthier processing cycles, more consumption of energy etc. Therefore, need arises for utilizing most advanced technology like Internet of Things (IOT) [1] to establish a comprehensive energy management platform which can effectively improve the efficiency of EMS, to achieve the prime goal of energy saving.IOT is a kind of network [2] which not only links the objects, can also capture the scientific management at any time through distinct transducer devices and the Internet. IOT can be fully automatic and can process information intelligently. In this paper an EMS is designed based on IOT technology to improve the EMS levels to achieve a target of energy saving. The EMS based on IOT can solve the problems of receiving, transmitting and saving the massive data in EMS by using digital instruments such as smart energy meter [3], communicating networks, software, databases.

\section{SYSTEM STRUCTURE}

IOT is a platform that enables devices to communicate with each other. The system architecture can be divided into three levels which are enlightened below:
$>$ Sensing Layer
$>$ Communication Interface module
$>$ IOT Control

The system architecture with all the necessary components enables to make smart decisions about which components / appliance to stock up based on real-time information picked up by sensing devices connected. This section describes the system architecture of energy monitoring system. 


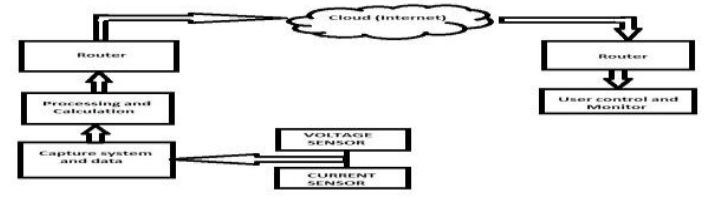

Fig.1. System architecture

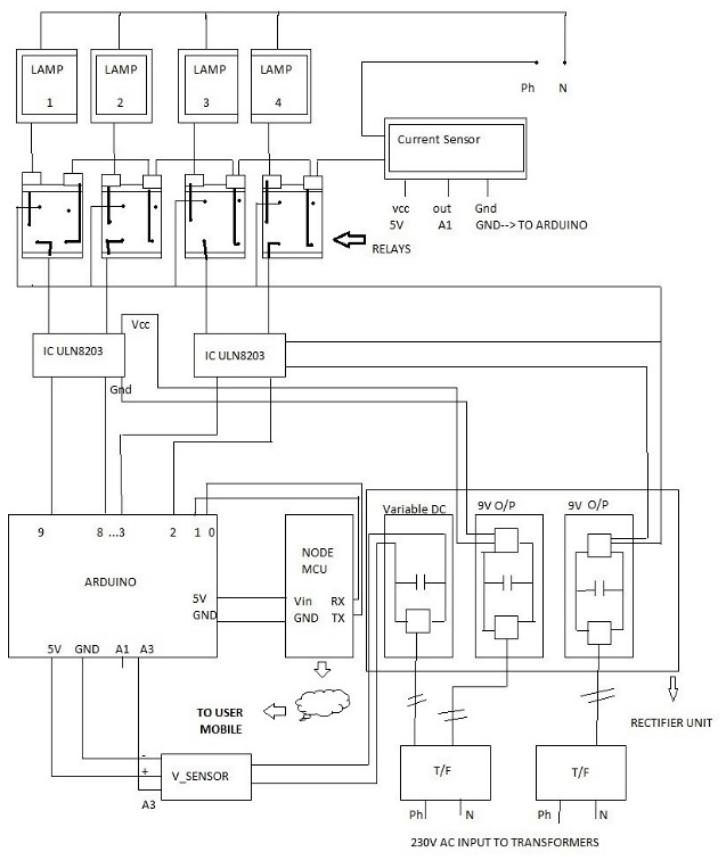

Fig.2.Block Diagram

\section{A. Sensing layer}

Electrical parameters like voltage, current are sensed using transducers (Sensors), that measures and converts the electrical quantity into a low voltage analog signal to ease measurement (like current, voltage, Power etc.) and can be read or interpreted by the controller unit. These devices act as inputs to the controller.

\section{Communication interface module}

The communication interface module is solely responsible in communicating with the IOT platform. They provide connectivity based on the wireless or wired communication protocol they are designed. They usually consist of embedded electronic modules that implement the communication.

Communication between IOT devices and the Internet are categorized as

a) Intermediate node acting as a gateway

(Internet-enabled).

b) Direct communication [4] to the Internet.

In the case "a", the IOT device is interfaced to the computer and transmits data via using a serial communication port. (USB). The received data from the internet is processed at the backend using appropriate software \& forwards it to the Internet. In the case (b), it adopts a simpler communication allowing devices to communicate more autonomously. In both the cases, packet data transformation is attained according to the wireless technology used like $\mathrm{Wi}-\mathrm{Fi}$, Bluetooth and ZigBee and through Ethernet using TCP/IP protocol.

\section{HARDWARE DESIGN}

Voltage Sensor:

The a.c input high voltage is to be reduced adopting available techniques like a potential divider or a converter that can be applied to the Arduino. The voltage limitation on the Arduino board is $5 \mathrm{~V}$ for analog signals. The voltage adapted in this project is a5:1 voltage divider network using 30k and a 7.5k resistors combined in series combination. A rugged voltage sensor shown in the Fig.3. is used in developing the model.

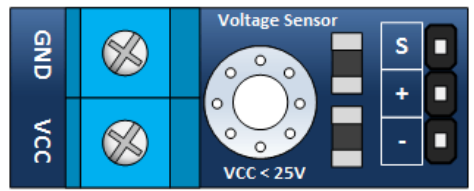

Fig.3 Voltage sensor

This module is based on resistance points pressure principle, and it can make the input voltage reduce 5 times of original voltage. Fig 4. Shows its connection diagram and response of the voltage sensor.

Specifications:

1 Voltage (input range) : DC $0-25 \mathrm{~V}$

2 Sensing range : DC $0.02445 \mathrm{~V}-25 \mathrm{~V}$

3 Resolution: $0.00489 \mathrm{~V}$

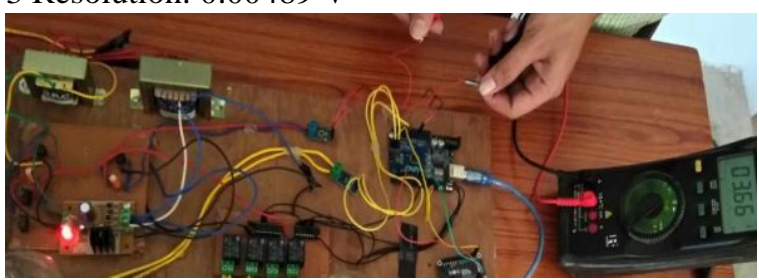

Fig. 4. Connection diagram and sensor output CURRENT SENSOR:

The input AC current is sensed and transformed into an voltage signal according to the magnitude of sensed input current using current sensor. The generated signal from the current signal is an analog signal and can be used to display the measured signal and interfaced to Arduino.

The current sensor chip ACS712ELC-05B is used in the present work. The module can measure plus or minus 5 amps, corresponding to the analog output of $185 \mathrm{mV} / \mathrm{A}$

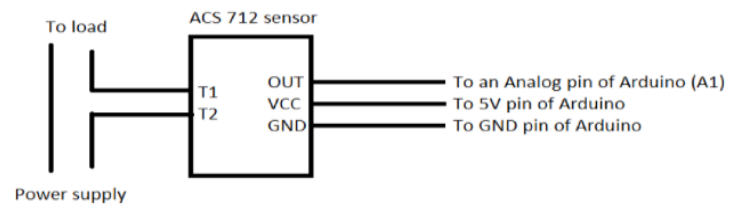

Fig. 5. Current sensor pin configuration

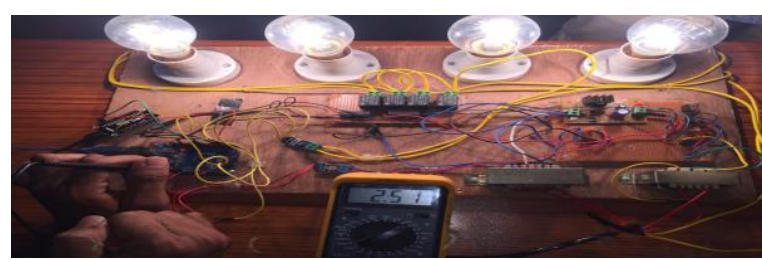

Fig. 6. Current sensor output 
Table I. Current sensor specifications

\begin{tabular}{|c|c|c|c|}
\hline $\begin{array}{c}\text { Specificat } \\
\text { ion }\end{array}$ & $5 \mathrm{~A}$ Module & $20 \mathrm{~A}$ Module & 30A Module \\
\hline $\begin{array}{c}\text { Supply } \\
\text { Voltage } \\
\text { (VCC) }\end{array}$ & $\begin{array}{c}5 \mathrm{Vdc} \\
\text { Nominal }\end{array}$ & $\begin{array}{c}5 \mathrm{Vdc} \\
\text { Nominal }\end{array}$ & $5 \mathrm{Vdc}$ Nominal \\
\hline $\begin{array}{c}\text { Measurem } \\
\text { ent Range }\end{array}$ & -5 to +5 & -20 to +20 & -30 to $+30 \mathrm{Amps}$ \\
\hline $\begin{array}{c}\text { Voltage at } \\
0 \mathrm{~A}\end{array}$ & $\mathrm{VCC} / 2$ & Amps & \\
& (nominally & (nominally & VCC/2 \\
(nominally \\
Scale & $185 \mathrm{Vdc}$ ) & $2.5 \mathrm{Vdc}$ ) & $2.5 \mathrm{VDC}$ ) \\
Factor & Amp & $100 \mathrm{mV}$ per & $66 \mathrm{mV}$ per Amp \\
\hline Chip & ACS712EL & ACS712ELC- & ACS712ELC-30 \\
& C-05A & $10 \mathrm{~A}$ & A \\
\hline
\end{tabular}

\section{Node MCU}

The Development Kit based on ESP8266, integrates GPIO, PWM, IIC, 1-Wire and ADC all in one board. Power your development in the fastest way communicating with NodeMcu Firmware. It has an software API for hardware IO, and can reduce the redundant work for configuring and manipulating hardware. it works with embedded Code used in Arduino. The term "NodeMcu" by default refers to the firmware rather than the dev kits. The firmware uses the Lua scripting language. It is based on the eLua project and built on the Espress if Non-OS SDK for ESP8266. It uses many open source projects, such as lua-cjson, and spiffs.

\section{IMPLEMENTATION \& DISCUSSION}

In the development of the system two software interfaces has been chosen one for programming the hardware (ARDUINO IDE) and another for mobile application development tool (BLYNK) for user interface.

The Arduino IDE is an open source which eases one to build, execute and dump the program into the Arduino hardware board. An embedded $\mathrm{C}$ has been used in building the code. Once the hardware has been initialized the function getVPP() receives the sensed data in to comport of the IDE where the received data can be viewed which is used further for calculating the power and energy values. These calculated values are further sent to the IOT cloud server and can be viewed on mobile device at the user end. The Blynk app helps in creating user interface with login screen for authenticated users, a display screen where the user choice for on/off button for device control, the amount of energy consumed, power rating, voltage and cur current drawn by an electrical appliance.
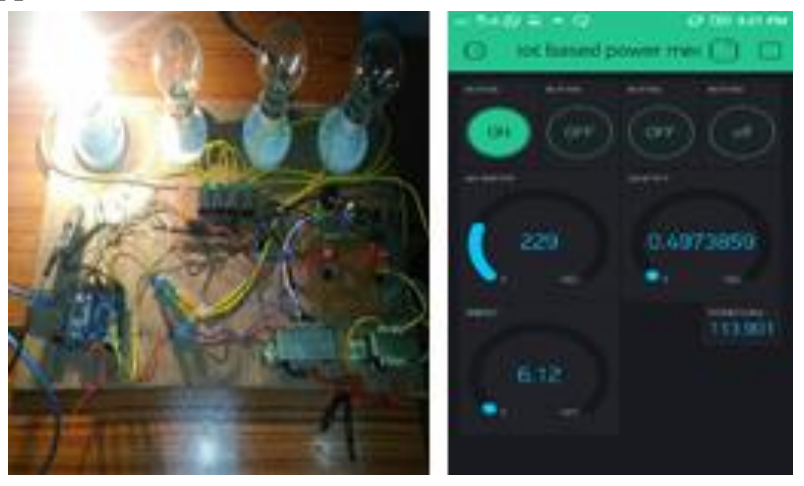

Fig.7 Device ON / OFF state indicator with display in mobile
Figure 7. indicates the on state of the electrical appliance and the mobile app shows the device state, energy and power consumption.
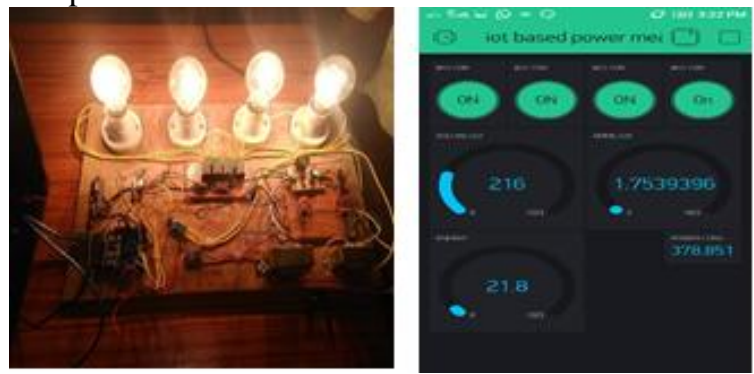

Fig.8 ALL Device ON / OFF state indicator with display in mobile

Similarly Fig.8. shows the control and measurement of four electrical devices. The user interface in the application enables to switch off any electrical appliance that is consuming more power and is not in use.

\section{CONCLUSION}

This paper presents the working of an efficient Energy measuring system that is capable of monitoring of electrical appliance and control of electrical energy based on IOT. This model enables us to observe the energy consumption during peak and off-peak states there by calculating the overall consumption by an appliance per day giving the user a choice to manage the energy consumption over a period. Finally, the implemented model demonstrated that it can control the appliances from the mobile. It is Easy to monitor the Voltage, Current, Power and Energy. Due to continuous monitoring we can reduce the wastage of energy and can be extended its use for in home appliances control, office and Industrial lighting load control. A limitation of this study, however, is the lack of available data that hinders the required empirical analysis on the benefits of IoT technology.

\section{REFERENCES}

1. P. P. Ray, "Internet of Things Cloud Based Smart Monitoring of Air Borne PM2 . 5 Density Level," in International conference on Signal Processing, Communication, Power and Embedded System (SCOPES), 2016, pp. 995-999

2. K. Siva, S. Ram, and A. N. P. S. Gupta, "IoT based Data Logger System for weather monitoring using Wireless sensor networks," Int. J. Eng. Trends Technol., vol. 32, no. 2, pp. 71-75, 2016.

3. YujunBao and Xiaoyan Jiang, "Design of electric Energy Meter for long-distance data information transfers which based upon GPRS", ISA2009. International Workshop on Intelligent Systems and Applications, 2009.

4. K. Li, J. Liu, C. Yue and M. Zhang, "Remote power management and meter-reading system based on ARM microprocessor", IEEE Precision Electromagnetic Measurements Digest CPEM, pp. 216-217, June, 2008

\section{AUTHORS PROFILE}

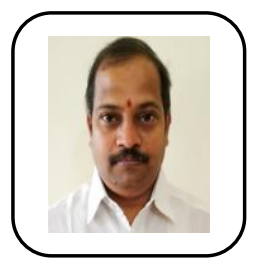

Vijaya anand Nidumolu is an Associate professor in the Department of Electrical and Electronics Engineering, P.V.P Siddhartha institute of technology, Vijayawada. His research areas of interest include Power system operation and control Soft computing technique and their application power engineering, IOT and Its applications

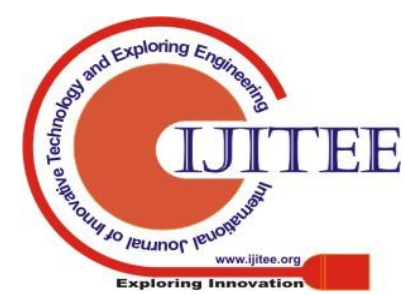




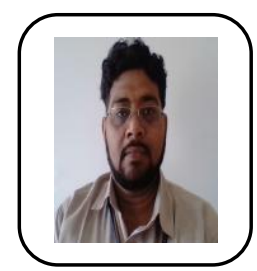

Venu Madhav $\mathbf{P}$ working as Assistant Professor in the Department of Electronics and Communication Engineering, P.V.P Siddhartha Institute of Technology, Vijayawada. His research areas of interest include design and development of IOT automation systems, antenna design and fabrication.

G.L.N.Murthy is a professor in the Department of Electronics and Communication Engineering, Lakireddy Bali Reddy College of Engineering , Mylavaram. His research areas of interest include Signal Processing, Image Processing and Bio medical Signal Processing, IOT and its applications. 